${ }^{\circledR}$ Entomologica Fennica. 29.VI.1990

\title{
A key to Diplonevra males of the Australasian and Oriental Regions, including two new species (Diptera, Phoridae)
}

\author{
R. H. L. Disney
}

Disney, R. H. L. 1990: A key to Diplonevra males of the Australasian and Oriental Regions, including two new species (Diptera, Phoridae). — Entomol. Fennica 1:33-39.

Recognition of the genus Diplonevra Lioy is clarified with respect to Dohrniphora Dahl. Dohrniphora bilineata Borgmeier is transferred to Diplonevra. A key to males of the Australasian and Oriental species is provided. Diplonevra ismayi sp. n., from Papua New Guinea, and D. predicta sp. n., from Australia, are described.

R. H. L. Disney, Field Studies Council Research Fellow, University Museum of Zoology, Downing Street, Cambridge, CB2 3EJ, England

In order to be in a position to describe two remarkable, termite-parasitizing, species of Diplonevra Lioy from Sulawesi (Disney 1986) it was necessary to construct a manuscript key to species of the Australasian and Oriental Regions. The discovery of two further new species has necessitated revision of this draft key, along with clarification of the distinction between Diplonevra and the closely related genus Dohrniphora Dahl.

This paper presents the revised key, along with descriptions of the new species, in order to provide the means of advancing knowledge of the biology of this interesting genus.

\section{Recognition of Diplonevra}

Diplonevra and Dohrniphora form a distinctive pair of genera within the Phorinae. The males can be recognised as follows: Wings fully developed, with a forked vein 3 (except in one species of Diplonevra) and normally developed costal cilia. Hind tibia with 1-3 longitudinal hair palisades. Palp two segmented. Supra-antennal bristles present.

The genera Myopiomyia Disney and Aenictomyia Brues are both only known in the female sex.
The former not only has hairs along vein 3 but on veins 4-6 as well. The latter has a single segmented palp and enlarged costal cilia.

Diplonevra gynaptera Fuller \& Lee was placed in a separate subgenus Apopteromyia by Beyer (1958) and subsequently Borgmeier (1967) gave as his "opinion" that it should be raised to generic rank. He advanced no reasons in support of this opinion. In the male sex the only distinctive feature is that the distal expansion of the apical section of vein 1 is greater than usual. The aberrant female can scarcely be regarded as adequate grounds for erecting a new genus in view of the fact that in this case the aberrations are failures to develop (e.g. wings and halteres) or incomplete developments (e.g. the reduced thorax and eyes). Such changes probably represent relatively small gene changes affecting the time of cessation of Juvenile Hormone secretion during development; as suggested as an explanation for the remarkable dimorphism in the females of Metopina ciceri Disney (1988a). There is a marked sexual dimorphism in the degree of wing development in Diplonevra mortimeri Disney, with the female wing being only about a third of the area of the male wing (figs 9 and 10 in Disney 1986). Furthermore, the females of many species are not known. 
I propose, therefore, to ignore Borgmeier's opinion.

The principal difference between the genus Dohrniphora Dahl and Diplonevra Lioy is universally agreed to be the presence of a single dorsal hair palisade on the hind tibia of the former and of two, or occasionally two and a half to three, such palisades in Diplonevra: in addition the mesopleuron is bare in Diplonevra but hairy in Dohrniphora (Borgmeier 1963, Disney 1983, Peterson 1987). Furthermore the form of the sensory complex at the base of the hind femur tends to be different in the two genera. A further distinction is in the form of the anal tube in males, such that the cerci originate near the apex in typical Diplonevra but at the base in Dohrniphora (figs 13 and 14 in Disney 1986). However, two curious species from Sulawesi were assigned to Diplonevra despite the form of the anal tube being more Dohrniphora-like, and likewise the sensory complex of the male hind femur (Disney 1986). However the interpretation advanced is fully supported by the condition in the new species from Australia, described below (Fig. 2A). It, like some other species of Diplonevra, lacks a sensory complex at the base of the hind femur.

Disregarding the consensus on the importance of the number of hair palisades and the hairing of the mesopleuron, Borgmeier (1961) described a Dohrniphora bilineata which, despite having two hair palisades on the hind tibia and a bare mesopleuron, he assigned to Dohrniphora. He never the less continued to endorse the consensus that this pair of characters allowed one to distinguish Diplonevra from Dohrniphora (Borgmeier 1963). In his assignment of $D$. bilineata to Dohrniphora, rather than Diplonevra, Borgmeier (1961) argued that the presence of a line of postero-dorsal hairs between the two hind-tibial hair palisades did not allow placement of the species in Diplonevra. He claimed that in the latter genus any postero-dorsals were always situated behind the posterior of the two hair palisades. This claim cannot be sustained. For example in Diplonevra peregrina (Wiedemann) there is a row of hairs between the two hair palisades, as well as postero-dorsal spines behind the posterior palisade. The base of the hind femur in D. bilineata (Borgmeier 1961, fig. 15) is certainly more Dohrniphora-like than Diplonevralike, but there is no absolute division into two distinct types among the great variety of forms to be found in the two genera. Borgmeier did not illustrate the hypopygium of $D$. bilineata, but he commented that the anal tube is "scheidenfoermig". This suggests it is of the Diplonevra type. I conclude that $D$. bilineata should be transferred to the genus Diplonevra, while noting that it seems to show some affinities with the two aberrant Sulawesi species (see Disney 1986). I formally transfer D. bilineata thus:

Diplonevra bilineata (Borgmeier) comb. $\mathrm{n}$. = Dohrniphora bilineata Borgmeier, 1961:19.

IIolotype \# New Guinea (Irian Jaya), Biak Island [not examined].

With the removal of $D$. bilineata from the genus Dohrniphora we can once again regard a single hair palisade on the hind tibia and a hairy mesopleuron as being diagnostic for this genus.

\section{Key to Diplonevra males}

Borgmeier (1967) provides a check list of Diplonevra species for the Australasian and Oriental Regions. To this must be added $D$. gynaptera Fuller \& Lee (1938), D. bilineata (Borgmeier, 1961) (see above), D. mortimeri Disney (1986) and $D$. watsoni Disney (1986). In addition two new species are described below.

This key has been put together largely from the literature. Names of species only known in the female sex are placed in square brackets. They have been included on the basis of characters not known to be sexually dimorphic in the genus. Identifications not supported by illustrations should be checked against their published descriptions, which are listed by Borgmeier (1968).

1. Hind tibia with at least one bristle before the apical quarter ............................................................. 2

- Hind tibia without pre-apical bristles ................... 13

2. Hind tibia with a single antero-dorsal bristle ......... 3

- Hind tibia with 2 or 3 antero-dorsal bristles ............ 8

3. Vein 3 unforked. Legs uniformly yellowish brown ... [dohrniphoroidea (Assmuth)]

- Vein 3 forked. Front and middle legs yellowish, as well as basal half of hind femora. Rest of hind legs darkly pigmented (though tibia may be a little paler than tarsus and apex of femur) 
4. Wing membrane usually strongly tinged, but if paler then vein 4 is nearly straight .....

- Wing membrane only lightly tinged with grey, and vein 4 (1st thin vein) is recurved (rearwards) in apical third .......................................... nigrita (Malloch)

5. Thorax largely yellowish with contrasting brown scutellum and postnotum. Hypopygium as Fig.2A, with elongated tergite 10 with well-developed surstyli. (Base of hind femur without sensory complex)

predicta $\mathrm{sp} . \mathrm{n}$.

- Thorax brown to almost black. Hypopygium otherwise .....

6. Abdomen with tergite 1 and anterior part of 2 yellow, contrasting sharply with dark brown to blackish thoracic dorsum and the rest of the abdominal tergites. (Base of hind femur without sensory complex) bifasciata (Walker)

- Abdominal tergites uniformly dark brown .............. 7

7. Anal tube somewhat compressed and clear pale yellow compressicauda Beyer

- Anal tube not compressed, and with a dark yellow distal half and even darker basal half ... lucida Beyer

8. With 3 antero-dorsal bristles on hind tibia ............. 9

- With only 2 antero-dorsals on hind tibia ............... 11

9. Hind tibia with 3 antero-ventral bristles in addition to antero-dorsals. (Basal half of hind femur as Fig. 3D) peregrina (Wiedemann)

- Hind tibia without a row of antero-ventral bristles 10

10. Knob of haltere yellow ........... [rufivenia Borgmeier]

- Knob of haltere blackish ............... [hyalizona Beyer]

11. Both basal and apical sections of vein 4 (1st thin vein) are slightly recurved (curved rearwards) 12

- Only apical section of vein 4 is slightly recurved ...... ........ [evanescens (Brues) and fasciiventris (Brues)] Note. The distinction between these two species requires elucidation.

12. Anal tube with distal half, or more, yellow ................ assmuthi (Schmitz)

- Anal tube yellowish but with distal half progressively darkened ............................................ varians Beyer

13. Scutum of thorax yellowish, contrasting with dark scutellum ........................ nigroscutellata (Malloch)

- Scutum and scutellum brown to dark brown ......... 14

14. Fore metatarsus with a darkened middle third (or more) contrasting with pale apical and basal thirds, and apical quarter to third of fore tibia at least a little darkened also. (Hypopygium as Fig. 2B. Sensory complex at base of hind femur as Fig. 3B) ......... watsoni Disney

- Fore tarsus and tibia more or less uniformly coloured. 15

15. Knob of haltere largely dark brown ..................... 16

- Knob of haltere largely yellowish ....................... 18

16. Apical section (from bend onwards) of vein 1 (R1) expanding so that distally it merges with costa along entire length of costal sections $2+3$ gynapter $a$ Fuller \& Lee

- Apical section of vein 1 only very slightly expanded distally

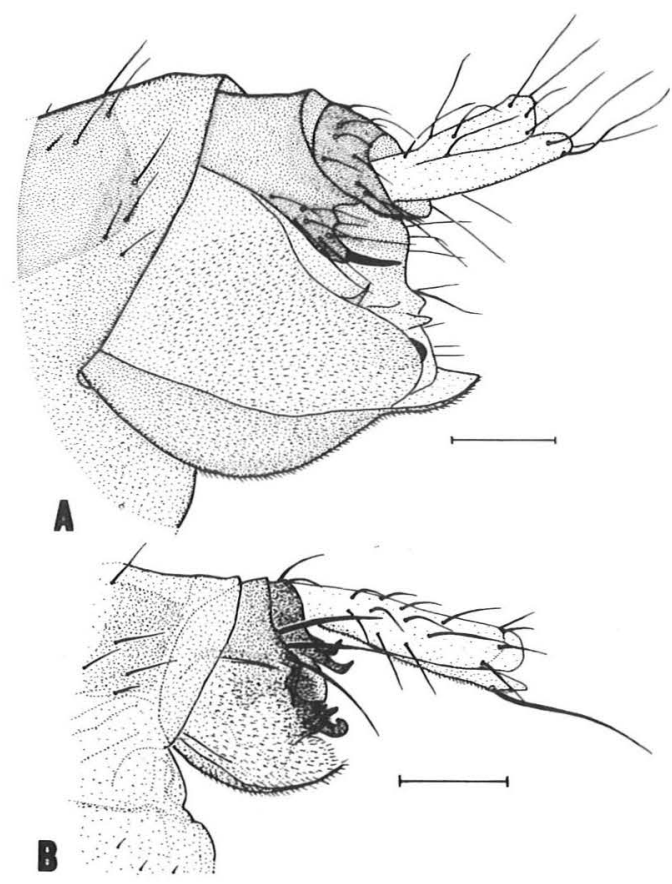

Fig. 1. Diplonevra male hypopygia viewed from left side. - A: D. ismayisp. n.; - B: D. mortimeri Disney. - Scale bars $=0.1 \mathrm{~mm}$.

17. Hypopygium as Fig.1B. (Sensory complex at base of hind femur as Fig. 3C) ................. mortimeri Disney

- Hypopygium with much longer, more slender, and more sinuous anal tube (fig. 6 in Borgmeier, 1967) .. caudata (Schmitz)

18. Legs all brown. Hypopygium as Fig. 1A. Sensory complex at base of hind femur as Fig. 3A ismayi sp. $\mathrm{n}$.

- Legs partly or entirely yellow. Hypopygium and sensory complex at base of hind femur otherwise ....... 19

19. Left side of epandrium with a single short bristle at rear... bilineata (Borgmeier)

- Left side of epandrium with about six bristles at rear (fig. 1 in Beyer, 1966) novaguineae Beyer

\section{Diplonevra ismayi sp. $\mathbf{n}$.}

Figs. 1A, 3A

Type material: Holotype \#, Papua New Guinea, Central P., nr. Eilogo, 24. VIII. 1981, rain forest (J. W. Ismay) (in Cambridge University Zoology Museum). Paratypes: $3 \#$ \#, 4aa same data as holotype except dates include 24 . VI. and 8. VIII. 1981. 
Diagnosis: As indicated in the key the male hypopygium and sensory complex at base of hind femur are distinctive in this species.

Male: Frons brown, darkening above. Median furrow not apparent. The single pair of supraantennals well developed. Antials close to, and about level with, antero-laterals and further apart than pre-ocellars. The latter slightly further apart than either is from a medio-lateral. The latter a little lower on frons. No bristle on cheek and a single bristle on jowl. The most ventral postocular bristle also enlarged. Third antennal segment brown, but not dark, and distinctly pointed. Arista short-haired. Palps orange to dusky yellowish with a darker basal segment. The apical segment with six apical bristles, the most basal being the longest, and with about eight hairs below basal half. Proboscis with palish labella, and smallish, brownish labrum.

Thorax brown throughout. Mesopleuron bare. Notopleuron with four bristles, but the second is weaker and shorter than the others. Scutellum with 4 bristles.

Abdomen with brown tergites with minute hairs only, except at rear of tergite 6 (Fig. 1A). Venter brownish, with a few hairs on segment 5 and 6. Hypopygium as Fig. 1A, being largely brown with a dirty yellow anal tube.

Legs with largely brown coxae and femora, otherwise yellowish. The hind tibia somewhat browner. Fore metatarsus no wider than following segments. Fore tibia with a short dorsal bristle at end of basal third and 6-9 short dorsal spines in apical half. Mid tibia with both dorsal and anterior longitudinal hair palisades running for most of length. The basal pair of bristles with the anterior one clearly shorter than the dorsal one. With two dorsal, longitudinal, hair palisades running the length of hind tibia, which lacks pre-apical bristles. Basal half of hind femur as Fig. 3A.

Wings $1.75-1.90 \mathrm{~mm}$ long. Costal index $0.51-0.53$. Costal ratios 5.90-7.44:0.86-1.59: 1 . Costal cilia $0.06 \mathrm{~mm}$ long. Vein 3 with a short hair at base. With four strong bristles on axillary ridge. Vein Sc only evident at extreme base. Veins brown, but vein 7 very pale. None of thin veins reach wing margin. Costal sections 2 and 3 a little thickened. Lumen of fork of vein 3 very small.
Membrane lightly tinged grey. Haltere largely yellowish, but a little darkened at apex of knob.

Female: Head similar to male but with following differences. Third antennal segment darker and less pointed. Outer face of palp with sensory excavation. Proboscis much longer and more massive; there being a long, broad, brown labrum and elongate labella bearing strong teeth on their inner faces.

Thorax as male. Abdomen with colouring as male, except ventrally the venter is a little paler, and also along the segment boundaries. The result is that the abdomen appears to be transversely banded when viewed from above. Only tergites 1-4 present, with 2 being widest and longest. Tergite 4 is represented by a small pigmented patch hardly broader than long. Tergites 5 and 6 represented by hairs only. Internally a chitinised, irregular ring-shaped furca (sternite 9 ) is evident in the atrium (genital chamber). Anal tube conspicuously haired and yellow.

Legs as male, but without sensory complex at base of hind femur, and fore tibia with 7-11 short dorsal spines in apical half.

Wings $2.03-2.18 \mathrm{~mm}$ long. Costal index $0.51-0.53$. Costal ratios 5.27-6.50:0.92-1.20:1. Costal cilia $0.06 \mathrm{~mm}$ long. Otherwise as male, and likewise the haltere.

\section{Diplonevra peregrina (Wiedemann)}

Fig. 3D

Phora peregrina Wiedemann, 1830:600

Phora sinensis Schiner, 1868:224

Phora conventa Brues, 1911:535

Dohrniphora setitibia Malloch, 1925:333

Material examined: \# Sulawesi, Dumoga-Bone National Park, Toraut Forest III. 1985 (Disney). \#\#, aa Japan, Tokyo 12. VII. 1976 (H. Kurahashi).

This widely distributed species is somewhat variable. Specimens from Japan, which I have examined, have longer and more numerous hairs adjacent to the two black spinules at the base of hind femur. A similar variation occurs in the related D.florea (Fabricius) (see figs 47 and 48 in Disney 1983). 


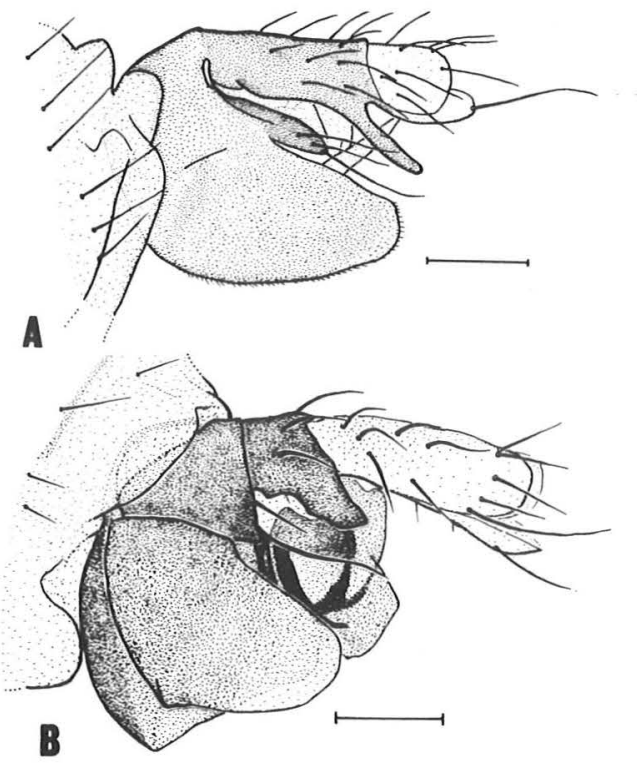

Fig. 2. Diplonevra male hypopygia viewed from left side. - A: D. predicta sp. n.;- B: D. watsoni Disney. - Scale bars $=0.1 \mathrm{~mm}$.

\section{Diplonevra predicta sp. $\mathbf{n}$.}

Fig. 2A

Type material: Holotype \#, AUSTRALIA: Queensland, Innisfail, Pin Gin Hill, 14-17 February 1983, in water trap (D. Howse) (In Australian Museum, Sydney, Australia). Paratypes: 5\# 10, same data as holotype except 4 deposited in University Museum of Zoology, Cambridge.

Diagnosis: As indicated in the key, this is a distinctive species in its colouring and male hypopygium.

Male: Frons brown, with darker pigment around each ocellus. Only a vague trace of median furrow in lower (anterior) half. The single, strong pair of supra-antennal bristles are situated on a forward, median extension of the frons. Antials only slightly closer together than either is from an antero-lateral bristle. The latter only slightly higher on frons. Pre-ocellars clearly further apart than antials, and a little further apart than either is from a medio-lateral bristle. The latter are a little lower on the frons. A single small bristle on each cheek but two long, strong bristles on each jowl. The third antennal segment is orange-brown, subspherical to slightly pointed, with a pre-apical, short- haired arista. Palp with a short pale brownish basal segment and a yellow, sub-cylindrical but basally-wider, distal segment with an apical cluster of about 6 robust black bristles. Proboscis largely yellowish, with a pale labrum which is no wider than a palp. Labella pale, with well-developed pseudotracheae below.

Thorax largely yellow, to more orange on top, contrasting with brown scutellum, postnotum and mid-coxae. Mesopleuron bare. Three notopleural bristles. Scutellum with a posterior pair of bristles and an anterior pair of strongish hairs.

Abdomen with pale yellow, hairless venter. Tergite 1 well developed but entirely yellow apart from the short dark hairs. Tergites 2-5 subequal, brown with paler, yellowish margins and minute scattered hairs. Tergite 6 longer, also brown with yellow margins, but with much longer hairs, especially postero-laterally. Hypopygium as Fig. 2A, being brown but with a somewhat paler epandrium and yellow cerci and proctiger.

Legs largely yellowish with darker tarsi, the hind tarsi being distinctly brown. In addition the hind tibiae are more dusky yellowish to pale brownish, and the dorsal edge and apical quarter of hind femur are brown. The fore metatarsus is somewhat stout. The fore tibia has a short dorsal bristle at end of basal third and 5-7 short dorsal spines in apical half. Mid tibia with a dorsal hair palisade extending its length and an antero-dorsal palisade in basal half. A strong pair of bristles (1 dorsal, 1 antero-dorsal) in basal quarter. Hind tibia with a full-length dorsal hair palisade and an antero-dorsal, slightly irregular, palisade running just over three-quarters length. An anterior to antero-dorsal bristle at end of first third. Only short hairs below basal half of hind femur and no sensory complex at base.

Wings $1.23-1.51 \mathrm{~mm}$ in length. Costal index $0.41-0.43$. Costal ratios 5.00-6.75: 0.73-1.23: 1 . Costal cilia $0.05 \mathrm{~mm}$ long. Vein 3 with a short hair at base. With one or, more usually, two subequal bristles on axillary ridge. Vein Sc very pale. Other veins pale brown, but vein 7 is obscure in basal half and all four thin veins fade away before reaching wing margin. Membrane lightly, but distinctly, tinged brownish grey. Halteres yellow.

Female: Head and thorax as male. Abdomen with tergites on segments $1-5$, being very wide on segment 2 but progressively narrowing to the 


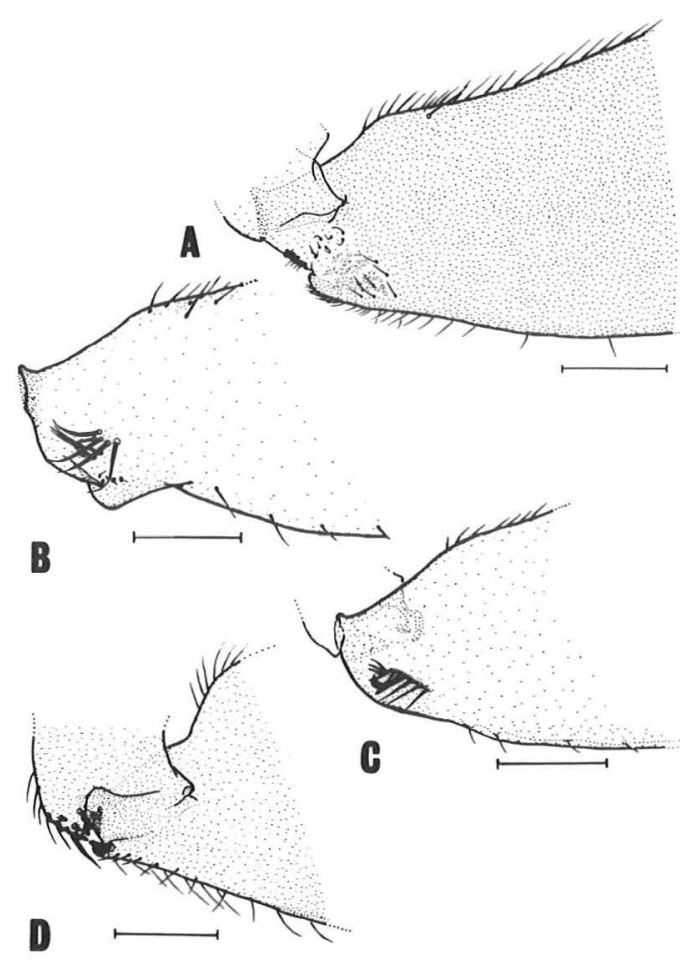

Fig. 3. Diplonevra males, bases of posterior face of hind femur. - A: D. ismayi sp. n.; - B: D. watsoni Disney; - C: D. mortimeri Disney; - D: D. peregrina (Wiedemann). - Scale bars $=0.1 \mathrm{~mm}$.

tapered tergite 5, which is about as long as width of anterior margin. Tergite 1 entirely yellow, the rest brown with yellow margins; and all have minute scattered hairs. The flanks below each tergite are brownish grey and segments 6 and 7 are almost entirely brownish grey. Otherwise venter is pale yellow, with a posterior transverse row of hairs on segment 6 and shorter hairs on rest of 6 and on segments 4 and 5 also. Legs as in male.

Wings $1.63-1.64 \mathrm{~mm}$ in length. Costal index 0.44 . Costal ratios $7.50: 1.24: 1$. Costal cilia 0.05 $\mathrm{mm}$ long. Otherwise as male.

\section{Diplonevra watsoni Disney}

Figs. 2B, 3B

Diplonevra watsoni Disney, 1986:414

The type series came from Sulawesi. Dr A. H. Kirk-Spriggs has sent me specimens from Maluku-
Utara, Bacan Island, Makian, $1.5 \mathrm{~km}$ East of Labula, 23-26. IX. 1985 (National Museum of Wales).

\section{Discussion}

The two new species possess male hypopygia which serve to reinforce the interpretation that the epandrium in Phoridae is indeed a true epandrium (tergite 9) and not a periandrium (Disney 1986, 1988b, 1990). In D. ismayi the fused tergite and sternite of segment 10 forms a cylindrical structure, bearing bristles, between the epandrium and the anal tube proper (Fig. 1A). In D. predicta this cylinder is elongated, but still retains the paired surstyli fused to the posterior ventro-lateral regions of the cylinder (Fig. 2A). If the D. ismayi condition of segment 10 were to be modified, by reduction and by loss of the bristles, then the postepandrial state would be as observed in Dohrniphora. If the D. predicta condition were to be modified, by loss of the surstyli and reduction of sclerotisation, then the result would be as observed in typical Diplonevra. Thus these two species represent two of the intermediate states predicted by the interpretation first given for the con-flies, D. mortimeri and D. watsoni, and the transformation series thereby postulated (Disney 1986).

\section{References}

Beyer, E. M. 1958: Bermerkungen uber zwei exotische Phoriden. - Broteria 27:121-123.

Borgmeier, T. 1961: Weitere Beitraege zur Kenntnis der neotropischen Phoriden, nebst Beschreibung einiger Dohrniphora-Arten aus der indo-australischen Region (Diptera, Phoridae). — Studia Entomol. 4:1-112.

- 1963: Revision of the North American Phorid flies. Part I. The Phorinae, Aenigmatiinae and Metopininae, except Megaselia (Diptera, Phoridae). - Studia Entomol. $6: 1-256$.

- 1967: Studies on Indo-Australian Phorid flies, based mainly on material of the Museum of Comparative Zoology and the United States National Museum (Diptera, Phoridae). — Studia Entomol. 9:129-326 (1966).

- 1968: A catalogue of the Phoridae of the world (Diptera, Phoridae). - Studia Entomol. 11:1-367.

Disney, R. H. L. 1983: Scuttle flies Diptera, Phoridae (except Megaselia). - Handbook Ident. British Insects 10(6):1-81. 
- 1986: Two remarkable new species of scuttle-fly (Diptera: Phoridae) that parasitize termites (Isoptera) in Sulawesi. - Syst. Entomol. 11:413-422.

- 1988a: A remarkable new species of scuttle fly (Diptera: Phoridae) whose larvae infest chickpea root nodules in India. - J. Nat. Hist. 22:611-616.

- 1988b: An interesting new species of Megaselia from Sulawesi, the ground plan of the Phoridae (Diptera) and phylogenetic implications for the Cyclorrhapha. Syst. Entomol. 13: 433-441.

- 1990: Phylogenetic implications of some features of Sulawesi scuttle flies (Diptera: Phoridae). - In: Knight, W. J. \& Holloway, J. D. (eds.), Insect and Rain Forests of South East Asia (Wallacea) - A special Project Wallace symposium. R. Entomol. Soc. London (in press).

Fuller, M. E. \& Lee, D. J. 1938: A new termitophilous Phorid (Diptera). — Proc. Linn. Soc. N. S. Wales 63:75-80.

Peterson, B. V. 1987: 51. Phoridae. — In: McAlpine, J. F. (ed.), Manual of Nearctic Diptera 2:689-712. - Research Branch, Agriculture Canada, Monograph No. 28. Ottawa, VI + pp. 675-1332.

Received 28.II.1989 